\title{
Transfusion of pooled buffy coat platelet components prepared with photochemical pathogen inactivation treatment: the euroSPRITE trial
}

\author{
Dick van Rhenen, Hans Gulliksson, Jean-Pierre Cazenave, Derwood Pamphilon, Per Ljungman, Harald Klüter, Hans Vermeij, \\ Mies Kappers-Klunne, Georgine de Greef, Michel Laforet, Bruno Lioure, Kathryn Davis, Stephane Marblie, Veronique Mayaudon, \\ Jocelyne Flament, Maureen Conlan, Lily Lin, Peyton Metzel, Don Buchholz, and Laurence Corash
}

\begin{abstract}
A nucleic acid-targeted photochemical treatment (PCT) using amotosalen $\mathrm{HCl}$ (S-59) and ultraviolet A (UVA) light was developed to inactivate viruses, bacteria, protozoa, and leukocytes in platelet components. We conducted a controlled, randomized, double-blinded trial in thrombocytopenic patients requiring repeated platelet transfusions for up to 56 days of support to evaluate the therapeutic efficacy and safety of platelet components prepared with the buffy coat method using this pathogen inactivation process. A total of $\mathbf{1 0 3}$ patients received one or more transfusions of either PCT test (311 transfusions) or conventional reference (256 transfusions) pooled, leukoreduced plate-
\end{abstract}

let components stored for up to 5 days before transfusion. More than $50 \%$ of the PCT platelet components were stored for 4 to 5 days prior to transfusion. The mean 1-hour corrected count increment for up to the first 8 test and reference transfusions was not statistically significantly different between treatment groups (13 $100 \pm 5400$ vs $14900 \pm 6200, P=.11$ ). By longitudinal regression analysis for all transfusions, equal doses of test and reference components did not differ significantly with respect to the 1-hour (95\% confidence interval [Cl], -3.1 to $6.1 \times 10^{9} /$ $\mathrm{L}, P=.53)$ and 24 -hour $(95 \% \mathrm{Cl},-1.3$ to $\left.6.5 \times 10^{9} / \mathrm{L}, \quad P=.19\right)$ posttransfusion platelet count. Platelet transfusion dose, pretransfusion storage duration, and patient size were significant covariates $(P<.001)$ for posttransfusion platelet counts. Clinical hemostasis, hemorrhagic adverse events, and overall adverse events were not different between the treatment groups. Platelet components prepared with PCT offer the potential to further improve the safety of platelet transfusion using technology compatible with current methods to prepare buffy coat platelet components. (Blood. 2003;101: 2426-2433)

ㄷ 2003 by The American Society of Hematology

\section{Introduction}

Despite continued improvements in pretransfusion donor screening and testing to detect viruses associated with transfusion-transmitted infections, blood components continue to carry risk of infectious disease, including bacteria. ${ }^{1}$ Nucleic acid detection methods for HIV and hepatitis $\mathrm{C}(\mathrm{HCV})$ have reduced the window period for infectious risk, but may not eliminate transfusion-associated $\mathrm{HIV}^{2}$ or $\mathrm{HCV}$ infections; ${ }^{3}$ fatal bacterial infections caused by transfusion of platelet concentrates continue to be reported. ${ }^{4}$ To improve the safety of platelet and plasma transfusion, a nucleic acid-targeted photochemical treatment (PCT) using the psoralen compound amotosalen $\mathrm{HCl}$ (S-59) and longwavelength ultraviolet A (UVA) light was developed to inactivate viruses, bacteria, and protozoa that may contaminate platelet and plasma components. ${ }^{5,6}$ Preclinical studies have demonstrated inactivation of more than $10^{6}$ infectious HIV particles, more than $10^{5}$ infectious hepatitis $\mathrm{B}(\mathrm{HBV})$ and $\mathrm{HCV}$ particles, and a broad spectrum of gram-positive and gram-negative bacteria. ${ }^{5,7,8}$ Furthermore, this process inactivates more than $10^{5}$ contaminating $T$ cells with effective inhibition of proliferation and complete suppression of leukocyte cytokine synthesis. ${ }^{9} 10$

To utilize this technology in blood component processing, a device consisting of a series of closed interconnected plastic containers and a microprocessor-controlled UVA light source has been integrated into a system for preparation of therapeutic doses of platelets. To evaluate the therapeutic efficacy and safety of platelet components prepared using this device, a randomized, controlled, double-blinded study was conducted on an intent-totreat (ITT) basis for patients who required repeated platelet transfusions during multiple periods of thrombocytopenia.

\section{Patients and methods}

\section{Patients and study design}

Patients with thrombocytopenia or receiving therapy expected to cause thrombocytopenia requiring platelet transfusion (suggested transfusion
From the Sanquin Blood Bank South West Region, Rotterdam, The Netherlands; Erasmus Medical Center, Rotterdam, The Netherlands; Huddinge University Hospital Stockholm, Sweden; Institute for Transfusion Science, Bristol, United Kingdom; Établissement Français du Sang EFS-Alsace, Strasbourg, France; Institute of Transfusion Medicine and Immunology, University of Heidelberg, Faculty of Clinical Medicine, Mannheim, Germany; University of Washington, Seattle; Baxter Healthcare Corp, Deerfield, IL; and Cerus Corp, Concord, CA.

Submitted March 25, 2002; accepted October 26, 2002. Prepublished online as Blood First Edition Paper, November 27, 2002; DOI 10.1182/blood-2002-03-0932.

A complete list of the members of the euroSPRITE investigator group appears

\section{in the "Appendix."}

Supported by Baxter Healthcare, Deerfield, IL, and Cerus, Concord, CA.

J.F., P.M., and D.B. are employees of Baxter Healthcare. M.C., L.L., and L.C. are employees of Cerus.

Reprints: Laurence Corash, Cerus Corp, 2411 Stanwell Dr, Concord, CA 94520; e-mail: larry_corash@ceruscorp.com.

The publication costs of this article were defrayed in part by page charge payment. Therefore, and solely to indicate this fact, this article is hereby marked "advertisement" in accordance with 18 U.S.C. section 1734.

(C) 2003 by The American Society of Hematology 
threshold of $<20 \times 10^{9}$ platelets/L) gave informed consent and were randomized to receive all required platelet transfusion support of the assigned treatment type, either test or reference, for up to 56 days of support. The inclusion criteria specified enrollment of patients 12 years or older admitted for treatment of acute or chronic leukemia (acute nonlymphocytic leukemia [ANLL], acute lymphocytic leukemia [ALL], chronic myelogenous leukemia [CML], chronic lymphocytic leukemia [CLL], or chronic myelomonocytic leukemia [CMML]), lymphoma, multiple myeloma, myelodysplasia, solid tumors, and hematopoietic stem cell transplantation. Patients with the following conditions at enrollment were excluded: splenomegaly $(>18 \mathrm{~cm}$ ), history of immune thrombocytopenia, disseminated intravascular coagulation, acute surgical condition, history of alloimmunization or refractoriness to platelet transfusion, pregnancy, or recent treatment with psoralen UVA (PUVA) therapy. Patients were enrolled at Bloodbank, Rotterdam, The Netherlands; Departments of Transfusion Medicine and Hematology, Huddinge University Hospital, Stockholm, Sweden; National Blood Service, Bristol, United Kingdom; and Établissement Français du Sang, Strasbourg, France. The study protocol was approved and informed patient consent was obtained in accordance with each study center's independent ethics committee. The study was conducted in accordance with European Council Directive 93/42/EEC, standard EN 540 1993, Good Clinical Practice Guidelines, International Committee on Harmonization Guidelines, and the Declaration of Helsinki.

The ITT population consisted of patients who were randomized and received at least one transfusion of test or reference platelet concentrate after randomization. All platelet transfusions were recorded. On-protocol transfusions consisted of platelet pools prepared according to treatment assignment. Off-protocol transfusions consisted of platelet products prepared by methods other than those specified in the study protocol. When platelet products of the assigned treatment type were unavailable, patients were transfused with conventional platelet products (off-protocol transfusions) according to the standard of care for each study center. Off-protocol platelet components were prepared by methods other than those specified in the protocol (single-donor apheresis and off-protocol random-donor methods) and samples were not taken to measure platelet dose or for culture because this was not standard practice at the study centers. Study centers were instructed to maintain an adequate inventory of study platelet products to minimize off-protocol platelet transfusion.

Primary care physicians ordered all platelet transfusions either for prophylaxis of bleeding or to treat active bleeding, per institutional standard of care, without knowledge of the patient's platelet treatment assignment. After completion of the 56-day transfusion period, patients were monitored for an additional 28 days to record adverse events. Following completion of this initial 84-day study period (cycle 1), patients were followed for need of additional platelet support while the study was open, or approximately one year, depending on study center. If additional platelet transfusion support was required, patients were asked to reregister for a second 56-day transfusion period and an additional 28-day adverse event surveillance period (cycle 2). Cycle 2 was conducted identically to cycle 1 , with the same treatment assignment for each patient.

\section{Platelet components}

Test platelet concentrates were prepared at each center as pooled buffy coats from 5 or 6 whole blood collections, using the Optipress device with top and bottom separation (Baxter Healthcare Corporation, La Chatre, France) and a platelet additive solution (InterSol; Baxter Healthcare Corporation, Lessines, Belgium) resulting in a final plasma concentration of approximately $35 \% .{ }^{11}$ Pooled platelet concentrates were leukoreduced by filtration followed by photochemical treatment (PCT) with $150 \mu \mathrm{M} \mathrm{S}-59$ and $3 \mathrm{~J} / \mathrm{cm}^{2}$ UVA treatment (Helinx Technology; Cerus Corporation, Concord, CA) for pathogen and leukocyte inactivation. ${ }^{5,8,9}$ After PCT, pooled platelet concentrates were stored for up to 5 days before transfusion. PCT was used in place of $\gamma$ irradiation for prevention of transfusion-associated graft-versus-host disease. ${ }^{10}$ Samples were obtained from test platelet pools at time of issue for transfusion to measure platelet content, $\mathrm{pH}$, and bacterial culture. The targeted platelet content of test pooled components was $3.0 \times 10^{11}$ platelets.

Reference platelet concentrates were prepared in conformance with standard operating procedures at each study center as pooled buffy-coat products from 4, 5, or 6 whole blood collections using the Optipress device with top and bottom separation process, conventional platelet pooling methods, and filtration leukoreduction. ${ }^{11}$ When feasible the number of buffy coats used to create a reference pool at each center was the same as that used for test pools. However, in one center (Bristol, United Kingdom), according to standard procedures, 4 buffy coats were used for preparation of conventional platelet pools, compared with 5 for test pools. In 2 study centers (Rotterdam and Stockholm) the reference platelet products were suspended in approximately $35 \%$ plasma and $65 \%$ platelet additive solution (T-Sol; Baxter Healthcare Corporation, Lessines, Belgium). ${ }^{11}$ In the other 2 centers (Bristol and Strasbourg) reference platelet pools were suspended in $100 \%$ donor plasma. Reference platelet pools were stored for up to 5 days prior to transfusion and were $\gamma$-irradiated per standard of care at time of issue per specific patient requirements for prevention of transfusionassociated graft-versus-host disease. Samples were obtained from reference platelet pools at time of issue for transfusion to measure platelet content, $\mathrm{pH}$, and bacterial culture. The targeted platelet content of reference pooled components was $3.0 \times 10^{11}$ platelets.

\section{Platelet transfusion}

Generally, a single pooled platelet component was transfused. If a second pooled platelet product was transfused within 4 hours of completion of the preceding platelet transfusion, the 2 pools were considered to be part of a single platelet transfusion episode. Platelet counts were measured from 10 minutes to 4 hours after platelet transfusion and from 18 to 24 hours after transfusion to determine the nominal 1- and 24-hour platelet count increments, respectively.

Off-protocol transfusions were given for the following indications: acute surgical conditions during which platelet count increments could not be accurately measured; refractoriness to platelet transfusion (with evidence of alloimmunization) requiring the use of single-donor human leukocyte antigen (HLA) matched or unmatched platelets; and lack of availability of on-protocol platelet products.

If a patient developed a temperature elevation of more than $2^{\circ} \mathrm{C}$ or more than $1^{\circ} \mathrm{C}$ with rigors within 24 hours after platelet transfusion, a blood culture was obtained and a reserved aliquot from the platelet component was cultured. Bacterial isolates obtained from positive blood cultures and platelet components were analyzed for identity to confirm the diagnosis of transfusion-transmitted bacteremia.

\section{Lymphocytotoxicity antibody assays and assay for potential S-59-related neoantigens}

Sera were collected and analyzed for lymphocytotoxicity antibodies at the Laboratory of Histocompatibility and Immunogenetics, Bloodbank Rotterdam, using an HLA-typed 60-cell panel. Sera reactive with $20 \%$ or more of cells in the panel were defined as reactive sera.

Sera were collected at enrollment and every 2 weeks during both transfusion cycles for detection of immunoglobulin $\mathrm{G}(\mathrm{IgG})$ antibodies directed against potential S-59-associated neoantigens. Test and reference group samples were analyzed, without knowledge of treatment assignment, using a microtiter plate enzyme-linked immunosorbent assay (ELISA; Cerus). Platelets from a pool of 12 donors were prepared with PCT or untreated, and $5 \times 10^{6}$ platelets per well served as the capture antigen. After overnight incubation $\left(2-8^{\circ} \mathrm{C}\right)$, plates were washed; blocked with IgGdepleted human serum; and test, negative control (pooled sera from 54 healthy donors), and positive control samples were added to the wells. Test samples and negative control samples were diluted 1:5. Positive controls of human anti-Pla1 serum (Immucor, Norcross, GA) were used at low (1:5) and high (1:50) dilutions. Plates containing samples were incubated for 1 hour at $37^{\circ} \mathrm{C}$ and washed. Fc-specific rabbit antihuman IgG was added and incubated, and plates were washed. Horseradish peroxidase conjugated to donkey antirabbit antibody was added and incubated; unbound conjugate was removed by washing. Human IgG bound to the target antigen was detected by measuring absorbance $(450 \mathrm{~nm})$ generated by tetramethylbenzidine substrate (Sigma-Aldrich, St Louis, MO). Samples were tested first against PCT-treated platelets. Samples that surpassed the cutoff value of the assay ( 2 SD beyond the reference range mean) were evaluated further by 
absorbing with untreated platelets followed by repeat assay with PCTtreated platelets. Samples that remained positive after absorption with untreated platelets were quantified for titer or relative concentration of IgG. Positive results for antibody to S-59-associated neoantigen required an optical density of $2 \mathrm{SD}$ beyond the reference mean value for normal sera with persistence following absorption with naive platelets. For positive samples, the relative concentration of the anti-PCT antibody was estimated by 2 methods. The assay was repeated with a trinitrophenol (TNP)-platelet/ goat anti-TNP/rabbit antigoat standard curve (analogous to the PCT-platelet/ human anti-PCT/rabbit antihuman complex in the assay). After the background subtraction from each format, the specific signal from the human sample was quantified in terms of the amount of goat antibody bound to TNP platelets yielding the same amount of signal. Also, positive antibody samples were diluted to estimate a relative measure of antibody titer. Two positive control samples containing antiplatelet antibodies (HPA-1) were used to characterize the sensitivity of the assay. These samples remained positive at a dilution of 1:4096. Quantitative IgG assay for these 2 samples using the TNP calibration standard indicated $\operatorname{IgG}$ concentrations of $9.8 \mathrm{ng} / \mathrm{mL}$ and $23.7 \mathrm{ng} / \mathrm{mL}$ for these 2 positive control sera.

\section{Study endpoints}

The 1-hour platelet count increment and the 1-hour corrected count increment $(\mathrm{CCI})$ were selected as coprimary endpoints because they are used in clinical practice to evaluate the efficacy of platelet transfusion. CCI was calculated using the platelet dose at time of issue for transfusion as follows: $\mathrm{CCI}=[$ (Posttransfusion count - Pretransfusion count $) \times$ Body surface area $\left.\left(\mathrm{m}^{2}\right)\right] /$ Platelet dose $\left(\times 10^{-11}\right)$.

Secondary study endpoints included the following parameters: the count increment and CCI 24 hours after platelet transfusion, the number of platelet transfusions during the period of platelet support, the interval between platelet transfusions, clinical hemostasis before and after platelet transfusion, the number of red cell units transfused during the period of platelet support, the proportion of patients with refractoriness to platelet transfusion (defined as 2 successive 1-hour CCIs $<5000$ ), and the proportion of patients with alloimmunization, defined as serologic conversion of the lymphocytotoxicity assay. Specific transfusion-related adverse events, including acute transfusion reactions, platelet transfusionassociated bacteremia, and all other adverse events were recorded, coded, and summarized using the MedDRA (Medical Dictionary for Regulatory Activities) preferred term synonym and the MedDRA system organ class. ${ }^{12}$

To determine the hemostatic efficacy of platelet transfusion, patients were evaluated within 6 hours before and for 6 hours after each platelet transfusion to assess bleeding. Twelve potential bleeding sites were evaluated by a blinded observer and assigned a score of 0 (no bleeding), 1 (minor bleeding), or 2 (major bleeding). ${ }^{13}$ The sites assessed were cutaneous, nasal, oropharyngeal, gastrointestinal, genital, urinary, pulmonary, retinal, and invasive sites (catheter, venipuncture, tracheostomy, surgical). For any bleeding site, if the severity grade was not recorded, the missing grade was estimated using the average score for other sites. The pre- and posttransfusion hemostatic scores were computed by summing the grades of the individual bleeding sites assessed before and after each transfusion. The mean score for all patients within each treatment group (test and reference) was computed. In addition to peritransfusion hemostatic scores, the protocol required reporting of all hemorrhagic adverse events during the active transfusion period and during the period of active surveillance. Hemorrhagic adverse events were graded for severity (nonserious and serious). All red cell transfusions during the active platelet transfusion period were recorded as a surrogate indicator of bleeding.

\section{Statistical rationale for study design}

The sample size and power calculations for this study were based on data for patients enrolled in the Trial to Reduce Alloimmunization to Platelets (TRAP) study. ${ }^{14}$ For 150 TRAP patients randomized to receive filtered single-donor platelets, the standard deviation of the mean of the first 8 CCIs was 5532, and the standard deviation of the mean of the first 8 count increments was $14.6 \times 10^{9} / \mathrm{L}$. For the present study, using a .05 -level 2-sided test, a sample size of 100 provided a power of $80 \%$ to detect a difference in mean CCI of 2807, and a difference in mean count increment of $8.0 \times 10^{9} / \mathrm{L}$ between patients receiving test and reference platelet components.

\section{Statistical methods}

Based on the TRAP experience, a majority of patients in this study were expected to receive at least 8 platelet transfusions during the 56-day transfusion period. To minimize the effect of variable numbers of transfusions, the mean 1- and 24-hour posttransfusion count increment and $\mathrm{CCl}$ were computed for up to the first 8 platelet transfusions during the 56-day transfusion period and compared by $t$ test. Comparisons were according to each patient's randomized treatment assignment.

To analyze platelet count increments for all transfusions, a stepwise linear regression model for longitudinal data using generalized estimating equations was used for all 1- and 24-hour posttransfusion platelet counts during the 56-day transfusion period of each cycle. ${ }^{15}$ Platelet transfusion number served as the longitudinal variable to deal with the effect of variation in the number of platelet transfusions among patients. The 1- and 24-hour posttransfusion platelet counts for all on-protocol transfusions were compared for test and reference patients with transfusion number, pretransfusion platelet count, platelet dose, average age of platelet concentrate, patient weight and height, and clinical site as covariates. The analysis was done stepwise with backward elimination of variables for which $P$ was more than .05, with retention of treatment (PCT or no PCT) at each step. Interactions with PCT for each significant covariate were added to the reduced model, and the process was repeated.

Analyses of the secondary endpoints were based on the ITT population with a significance level of .05 for the secondary analyses. No adjustment for multiplicity was made.

\section{Results}

\section{Patient population}

Between June 1998 and June 2000, 103 patients (52 test and 51 reference) received at least one study transfusion. More test patients $(83 \%)$ than reference patients $(67 \%)$ completed cycle 1 $(P=.06)$, and more test patients (10 of 52) than reference patients (2 of 51) received transfusions during cycle 2 . This difference resulted in a greater number of study observation days for test patients than for reference patients (4081 vs 3633 days).

The groups were balanced with respect to primary diagnoses, therapy, previous exposure to allogeneic blood products, and previous pregnancy (Table 1) and demographic characteristics (Table 2). Baseline hematology and coagulation profiles were

Table 1. Disease, therapy, and previous alloimmunization exposure of patients receiving platelet transfusions for thrombocytopenia

\begin{tabular}{lcr}
\hline & \multicolumn{2}{c}{$\begin{array}{c}\text { Treatment group, } \\
\text { no. }(\%)\end{array}$} \\
\cline { 2 - 3 } & $\begin{array}{c}\text { Test } \\
(n=52)\end{array}$ & $\begin{array}{r}\text { Reference } \\
(\mathrm{n}=51)\end{array}$ \\
\hline Primary diagnosis & & \\
Acute leukemia & $26(50)$ & $24(47)$ \\
Hematopoietic tumor*/nonhematopoietic solid tumor & $21(40)$ & $19(37)$ \\
Other & $5(10)$ & $8(16)$ \\
Therapy & & $1(2)$ \\
Bone marrow transplantation & $2(4)$ & $18(35)$ \\
$\quad$ Peripheral blood stem cell transplantation & $17(33)$ & $32(63)$ \\
$\quad$ Chemotherapy & $33(63)$ & $44(86)$ \\
Alloimmunization exposure & $46(88)$ &
\end{tabular}

There were no statistical differences between groups $(P>.05)$.

*Includes multiple myeloma, lymphoma, and Hodgkin lymphoma.

†Previous platelet or red blood cell transfusion or, for women, previous pregnancy. 
Table 2. Demographic characteristics of patients receiving platelet transfusions for thrombocytopenia

\begin{tabular}{lcc}
\hline & \multicolumn{2}{c}{ Treatment group, no. (\%) } \\
\cline { 2 - 3 } & Test, $\mathrm{n}=52$ & Reference, $\mathrm{n}=51$ \\
\hline Sex, no. (\%) & $30(58)$ & $28(55)$ \\
$\quad$ Male & $22(42)$ & $23(45)$ \\
$\quad$ Female & $48.6 \pm 14.1$ & $51.1 \pm 13.2$ \\
Age, mean $\pm \mathrm{SD}, \mathrm{y}$ & $73.56 \pm 15.30$ & $74.25 \pm 15.69$ \\
Weight, mean $\pm \mathrm{SD}, \mathrm{kg}$ & $173.09 \pm 8.70$ & $171.47 \pm 8.19$ \\
Height, mean $\pm \mathrm{SD}, \mathrm{cm}$ & $1.88 \pm 0.23$ & $1.89 \pm 0.22$ \\
BSA, mean $\pm \mathrm{SD}, \mathrm{m}^{2}$ &
\end{tabular}

There were no statistical differences between groups $(P>.05)$.

reflective of patients with neoplastic diseases requiring platelet transfusion (Table 3), and not different between groups. While on study, 102 patients (99\%) were taking some form of anti-infective, $26(25 \%)$ were exposed to amphotericin, $34(33 \%)$ were treated with anticoagulants, $4(4 \%)$ received fibrinolytics, $5(5 \%)$ received antifibrinolytics, and $2(2 \%)$ received nonsteroidal anti-inflammatory drugs (NSAIDs). There were no differences in concomitant medications between the treatment groups except for the use of antifibrinolytics; all 5 patients on this therapy were among patients assigned to reference transfusions $(P=.03)$.

\section{Platelet transfusions}

During cycle 1, 311 (80\%) of 390 test transfusions and $256(90 \%)$ of 286 reference transfusions were prepared according to protocol. Patients assigned to test received a mean of $7.5 \pm 5.8$ platelet transfusions, inclusive of on- and off-protocol transfusions, compared with $5.6 \pm 5.5$ for reference patients $(P=.09)$. The average number of on-protocol transfusions for test patients was $6.2 \pm 4.2$, compared with $5.0 \pm 4.8$ for reference patients $(P=.22)$. The average platelet dose $\left(10^{11}\right)$ per transfusion of on-protocol transfusions was lower for test transfusions than for reference transfusions $(3.9 \pm 1.0$ vs $4.3 \pm 1.2, P<.001)$. The mean total platelet dose of on-protocol transfusions for both treatment groups was similar (test mean total dose $22.3 \times 10^{11}$ vs reference mean total dose $\left.21.2 \times 10^{11} ; P=.74\right)$. Two factors contributed to the reduced platelet content of test platelet products: extra samples $(10 \mathrm{~mL})$ required to measure $S-59$ levels and loss due to platelet retention $(20 \mathrm{~mL})$ during transfers with use of a prototype treatment set. The average storage duration of test pools was comparable to that of reference pools $(3.5 \pm 1.1$ vs $3.4 \pm 1.2$ days; $P=.28)$, with median pretransfusion storage durations of 4 days for test and 3 days for reference platelet pools. Moreover, $22 \%$ of test pools and $20 \%$ of reference pools were transfused on the fifth day of storage. All test and reference platelet products were $\mathrm{ABO}$ compatible.

\section{Platelet count increments 1 hour after transfusion}

The mean 1-hour count increment for up to the first 8 transfusions was $27.5 \pm 13.5 \times 10^{9} / \mathrm{L}$ for test patients and $35.8 \pm 23.3$ for reference patients. The mean difference of $8.3 \times 10^{9} / \mathrm{L}(95 \%$ confidence interval $[\mathrm{CI}], 0.9-15.8)$ was statistically significantly different $(P=.03)$. Both test and reference platelet count increments were within reported therapeutic ranges. ${ }^{16,17}$ When the 1-hour count increment was adjusted for differences in platelet dose using the CCI, the mean 1-hour CCI was not statistically significantly different between treatment groups (13 100 \pm 5400 vs $14900 \pm 6200, P=.11)$, with a mean difference of $1800(95 \%$ CI, -400 to 4100$)$. By longitudinal regression analysis for all transfusions, platelet concentrates prepared with and without PCT did not differ significantly with respect to the 1-hour posttransfusion platelet count in cycle $1(P=.53)$. The estimated effect of PCT treatment was a decrease in the 1-hour posttransfusion platelet count of $1.5 \times 10^{9} / \mathrm{L}\left(95 \% \mathrm{CI},-3.1\right.$ to $\left.6.1 \times 10^{9} / \mathrm{L}\right)$.

Covariates with a significant $(P<.05)$ effect on the posttransfusion platelet count were, in descending order of magnitude, as follows: platelet dose $(P<.001)$, storage duration of the platelet concentrate prior to transfusion $(P<.001)$, pretransfusion platelet count $(P<.001)$, and patient weight $(P<.001)$. None of these covariates had significant interactions with PCT, including the interaction term for PCT treatment and dose $(P=.73)$. By regression analysis for all transfusions, PCT-treated and non-PCTtreated platelets gave comparable 1-hour posttransfusion platelet counts for equal platelet doses in cycle 1 (Figure 1). There were no significant differences among study sites in the magnitude or the direction of the effect of PCT on the count increment $(P=.80)$.

\section{Platelet count increments $\mathbf{2 4}$ hours following transfusion and the interval to the next platelet transfusion}

The mean 24-hour posttransfusion count increment (for up to the first 8 transfusions) for the test group $\left(16.4 \pm 9.5 \times 10^{9} / \mathrm{L}\right)$ was less $(P=.004)$ than for the reference group $\left(24.7 \pm 17.6 \times 10^{9} / \mathrm{L}\right)$. The mean 24-hour posttransfusion CCI (for up to the first 8 transfusions) was less $(P=.02)$ for the test group $(7400 \pm 5500)$ than for the reference group $(10600 \pm 7100)$. By longitudinal regression analysis for all cycle 1 transfusions, PCT platelets and non-PCT platelets did not differ significantly with respect to the 24-hour platelet count in cycle $1(P=.19)$. The estimated effect of PCT treatment was a decrease in the 24-hour platelet count of $2.6 \times 10^{9} / \mathrm{L}\left(95 \% \mathrm{CI},-1.3\right.$ to $\left.6.5 \times 10^{9} / \mathrm{L}\right)$. The same covariates were significant as for the analysis of 1-hour platelet count, and none of these covariates had significant interactions with PCT. The interaction term for PCT treatment and dose was not significant

Table 3. Baseline hematology and coagulation profiles of patients receiving platelet transfusions for thrombocytopenia

\begin{tabular}{|c|c|c|c|c|}
\hline & \multicolumn{4}{|c|}{ Treatment group } \\
\hline & \multicolumn{2}{|c|}{ Test, $\mathrm{n}=52$} & \multicolumn{2}{|c|}{ Reference, $\mathrm{n}=51$} \\
\hline & No.* & Value & No.* & Value \\
\hline Hematocrit, $\%$, mean \pm SD & 47 & $0.27 \pm 0.05$ & 50 & $0.26 \pm 0.05$ \\
\hline Platelet count, mean $\pm \mathrm{SD}, \times 10^{9} / \mathrm{L}$ & 52 & $19.1 \pm 13.3$ & 51 & $16.7 \pm 13.1$ \\
\hline Hemoglobin level, mean $\pm S D, g / L$ & 51 & $94.4 \pm 15.8$ & 48 & $89.0 \pm 19.1$ \\
\hline Fibrinogen level, mean $\pm \mathrm{SD}, \mathrm{g} / \mathrm{L}$ & 47 & $4.673 \pm 1.647$ & 44 & $4.724 \pm 1.203$ \\
\hline White blood cell count, mediant, $\times 10^{9} / \mathrm{L}$ & 52 & 0.350 & 51 & 0.220 \\
\hline Prothrombin time, mean $\pm S D$, sec & 28 & $12.8 \pm 4.1$ & 26 & $12.1 \pm 1.8$ \\
\hline Partial thromboplastin time, mean $\pm S D$, sec & 50 & $35.0 \pm 9.2$ & 46 & $34.7 \pm 9.2$ \\
\hline
\end{tabular}

There were no statistical differences between groups $(P>.05)$

*Number of patients with baseline values.

†The median is given, rather than the mean, owing to a nonnormal distribution of values. 


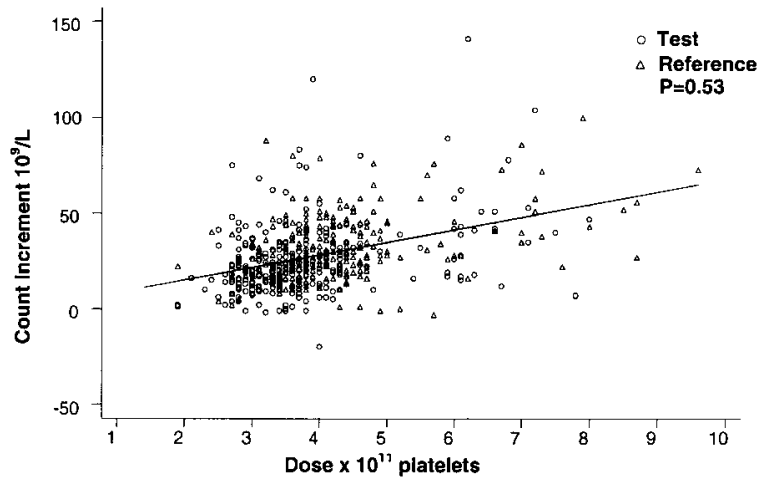

Figure 1. Effect of platelet dose on the 1-hour posttransfusion platelet count increment for test and reference platelet transfusions. The 1-hour posttransfusion count increment vs platelet transfusion dose is shown for all on-protocol (test and reference) transfusions in cycle 1 . Equal doses of test and reference platelet products resulted in comparable 1 -hour count increments $(P=.53)$ over the range of platelet doses transfused. The regression lines for test and reference platelet transfusions appear superimposed.

$(P=.31)$. By regression analysis for all transfusions, PCT-treated and non-PCT-treated platelets gave comparable 24-hour posttransfusion platelet counts for equal doses in cycle 1 (Figure 2).

Because 2 study centers suspended reference components in $100 \%$ plasma and 2 used a mixture of plasma and T-Sol, we compared the 1-hour and 24-hour count increment and CCI responses for this variable within the reference patient group. No differences were observed, consistent with previously reported experience using T-Sol-plasma mixtures. ${ }^{18}$

There was no statistically significant difference between test and reference populations with respect to duration of the first period of platelet support or total duration of platelet support (Table 4). The average interval between platelet transfusions was computed for the first period of platelet transfusion, because all patients $(n=103)$ underwent the first period of platelet support, and it was not significantly different for test and reference patients (Table 4).

\section{Posttransfusion platelet counts for patients enrolled in cycle 2}

Because of the limited numbers of patients enrolled in cycle 2 (10 test and 2 reference patients), data for posttransfusion platelet counts were analyzed only by longitudinal regression. The treatment groups did not differ significantly with respect to the 1-hour platelet count $(P=.47)$. The estimated effect of PCT was an increase in the 1-hour platelet count of $4 \times 10^{9} / \mathrm{L}(95 \% \mathrm{CI},-7$ to $\left.14 \times 10^{9} / \mathrm{L}\right)$. The interaction terms for PCT and dose were not significant $(P=.74)$. Similarly, the treatment groups did not differ significantly with respect to the 24-hour platelet count in cycle 2 $(P=.90)$. The estimated effect of PCT was an increase in the platelet count of $1 \times 10^{9} / \mathrm{L}\left(95 \% \mathrm{CI},-10\right.$ to $\left.11 \times 10^{9} / \mathrm{L}\right)$. PCTtreated and non-PCT-treated platelets provided comparable 1- and 24-hour platelet counts for equal doses in cycle 2.

\section{Bleeding, hemostatic scores, and red cell transfusions}

Based on peritransfusion hemostatic assessments, $71 \%$ of test and $63 \%$ of reference patients had at least one episode of bleeding before transfusion $(P=.36)$. The incidence of bleeding events was lower and similar in both groups after platelet transfusion (54\% test and $49 \%$ reference; $P=.62$ ). There were no significant differences in hemostatic scores between test and reference patients either before or after transfusion, and average hemostatic scores were lower after transfusion in both groups (Table 5). When hemostasis during the entire transfusion period was assessed on the basis of reported hemorrhagic adverse events, a high and equal proportion of patients had at least one hemorrhagic adverse event (Table 6). The most frequent hemorrhagic adverse events were epistaxis ( $42 \%$ test vs $41 \%$ reference), gingival bleeding (17\% test vs $12 \%$ reference), injection site hemorrhage (17\% test vs $6 \%$ reference), purpura ( $15 \%$ test vs $14 \%$ reference), petechiae (13\% test vs $16 \%$ reference), and hematoma (13\% test vs $8 \%$ reference). Only $6 \%$ of patients in each group experienced severe hemorrhagic adverse events (Table 6). Bleeding also was assessed indirectly by comparing the number of red blood cell units transfused during the transfusion periods. The average number of red blood cell units transfused during the first period of platelet support in cycle 1 (test $=4.9 \pm 4.2$, reference $=4.5 \pm 5.4$ ) was not different between groups $(P=.68)$. Additionally, the average number of red cell units transfused during the 56 days of cycle 1 (test $=9.3 \pm 5.3$, reference $=8.2 \pm 6.3$ ) did not differ significantly between treatment groups $(P=.34)$. The average number of red blood cell units transfused per day of platelet support during the first period of platelet support in cycle 1 (test $=0.41 \pm 0.35$, reference $=0.56 \pm 0.68)$ did not differ significantly between treatment groups $(P=.16)$.

\section{Refractoriness to platelet transfusion, lymphocytotoxic antibodies, and antibodies to potential S-59-associated neoantigens}

Patients were classified as refractory to platelet transfusions if the 1-hour CCI was less than 5000 for 2 successive transfusions. All patients diagnosed as refractory were evaluated for lymphocytotoxic antibodies (LCAs). Seven patients-4 (8\%) assigned to test and $3(6 \%)$ assigned to reference $(P=.72)$-had at least one refractory episode to platelet transfusion during the study. Of these 7 patients, 2 from the test group and 1 from the reference group tested positive for LCAs at baseline. One patient, who received test platelet transfusions, had a positive LCA result at baseline and remained refractory throughout cycle 1 . Two additional patients (one test and one reference) became LCA-positive during cycle 1 . One refractory patient in each group remained LCA-negative. No patient had confirmed antibodies directed against potential S-59related platelet neoantigens.

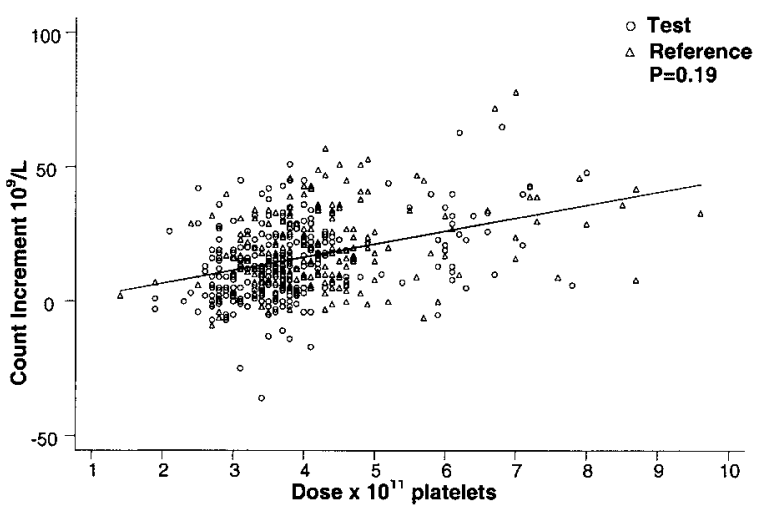

Figure 2. Effect of platelet dose on the 24-hour posttransfusion platelet count increment for test and reference platelet transfusions. The 24-hour posttransfusion count increment vs platelet transfusion dose is shown for all on-protocol (test and reference) transfusions in cycle 1 . Equal doses of test and reference platelet products resulted in comparable 24-hour count increments $(P=.19)$ over the range of platelet doses transfused. The regression lines for test and reference platelet transfusions appear superimposed. 
Table 4. Total duration of platelet support, duration within each period of platelet support, and transfusion interval in cycle 1

\begin{tabular}{|c|c|c|c|}
\hline & \multicolumn{2}{|c|}{ Treatment group } & \multirow[b]{2}{*}{$P$} \\
\hline & Test $(n=52)$ & Reference $(n=51)$ & \\
\hline Total duration of platelet support, mean $\pm S D, d$ & $15.2 \pm 11.2$ & $12.7 \pm 12.4$ & .29 \\
\hline \multicolumn{4}{|l|}{ Duration of platelet support by period within cycle 1 , mean $\pm S D, d$} \\
\hline Period 1 (no. of patients) & $11.7 \pm 9.5(52)$ & $10.1 \pm 11.7(51)$ & $\geq .45$ \\
\hline Period 2 (no. of patients) & $7.5 \pm 7.2(22)$ & $8.0 \pm 7.9(14)$ & \\
\hline Period 3 (no. of patients) & $15.0(1)$ & $5.0 \pm 6.9(3)$ & \\
\hline Time between transfusions during first period of platelet support, mean $\pm S D, d$ & $3.0 \pm 1.23$ & $3.4 \pm 1.21$ & .13 \\
\hline
\end{tabular}

All transfusions for the ITT population were recorded during the 56-day transfusion period. The period of platelet support was defined as the number of days from the first study transfusion to the last platelet transfusion. If an interval of 7 or more days without platelet transfusions occurred, the last platelet transfusion before the interval with no platelet transfusion was treated as the last platelet transfusion for that period of support. If platelet transfusions were resumed, the length of subsequent periods of platelet support was added to the first period of platelet support to determine the total period of platelet support during the 56-day transfusion cycle. Thus, patients could have multiple periods of platelet transfusion during a 56-day cycle.

\section{Acute transfusion reactions and other adverse events}

All patients were evaluated following platelet transfusion for transfusion-related symptoms and signs. Posttransfusion events classified as acute transfusion reactions included fever, chills, nausea, skin rash, urticaria, bronchospasm, tachycardia, hypotension, hypertension, hemoglobinuria, hemolysis, and change in vital signs within 6 hours following platelet transfusion. In cycle 1, 6\% of test transfusions were associated with acute reactions, compared with $5 \%$ of reference platelet transfusions $(P \geq .61)$.

A total of 147 types of adverse events were reported in $5 \%$ or more of the patient population evaluated during cycle 1 and cycle 2 . Overall, there were no differences in the incidence of adverse events by system organ class between the 2 treatment groups. Serious adverse events were reported in 14 (27\%) patients randomized to the test platelet treatment group and in 13 (25\%) patients randomized to the reference platelet group. Adverse events reported as related to platelet transfusion were not statistically significantly different between the groups $(P=.20)$. No study transfusions demonstrated transfusion-associated bacteremia. Four test patients and 5 reference patients died while on study. No deaths were related to study transfusions.

\section{Discussion}

The duration of platelet support and the demographic characteristics of the patient population in this study provided a meaningful transfusion experience to evaluate the efficacy of platelet components prepared with a pathogen inactivation process. This study used pooled random-donor platelets prepared with the buffy coat process, a methodology commonly used in Europe. ${ }^{19}$ Therapeutic platelet doses of test and reference products were pooled, filtered to reduce leukocyte content, and stored for up to 5 days prior to transfusion. Previous studies have shown that buffy coat platelets

Table 5. Pre- and posttransfusion hemostatic scores ${ }^{*}$ in cycle 1

\begin{tabular}{lccc}
\hline & \multicolumn{2}{c}{ Treatment group } & \\
\cline { 2 - 4 } & $\begin{array}{c}\text { Test } \\
(\mathrm{n}=52)\end{array}$ & $\begin{array}{c}\text { Reference } \\
(\mathrm{n}=51)\end{array}$ & $P$ \\
\hline Pretransfusion score & $0.43 \pm 0.46$ & $0.45 \pm 0.57$ & .84 \\
Posttransfusion score & $0.28 \pm 0.39$ & $0.30 \pm 0.43$ & .81 \\
\hline
\end{tabular}

Twelve potential bleeding sites were evaluated by a blinded observer and assigned a score of 0 (no bleeding), 1 (minor bleeding), or 2 (major bleeding). The pre- and posttransfusion hemostatic scores for each transfusion were computed by summing the grades of the individual bleeding sites before and after each transfusion.

${ }^{*}$ Mean \pm SD. and platelets prepared by the platelet-rich-plasma (PRP) process provided similar 1 - and 24-hour count increments. ${ }^{20}$ Similar observations have been reported for platelets collected by apheresis and suspended in $100 \%$ plasma. ${ }^{21}$ In addition, we, and others have observed that reference platelets suspended in $100 \%$ plasma and in mixtures of $35 \%$ plasma and $65 \%$ platelet additive solution (T-Sol)

Table 6. Hemorrhagic adverse events by system organ class in cycle 1

\begin{tabular}{|c|c|c|c|c|}
\hline \multirow[b]{2}{*}{ System organ class } & \multicolumn{2}{|c|}{$\begin{array}{c}\text { Test } \\
(\mathrm{n}=52), \text { no. }(\%)\end{array}$} & \multicolumn{2}{|c|}{$\begin{array}{c}\text { Reference } \\
(\mathrm{n}=51), \text { no. }(\%)\end{array}$} \\
\hline & Total & Severe & Total & Severe \\
\hline Any hemorrhagic event & $41(79)$ & $3(6)$ & $38(79)$ & $3(6)$ \\
\hline Eye disorders & $7(13)$ & $1(2)$ & $0(0)$ & $0(0)$ \\
\hline Eye hemorrhage & $3(6)$ & $0(0)$ & $0(0)$ & $0(0)$ \\
\hline Retinal hemorrhage & $3(6)$ & $1(2)$ & $0(0)$ & $0(0)$ \\
\hline Gastrointestinal disorders & $18(35)$ & $0(0)$ & $14(27)$ & $1(2)$ \\
\hline Gingival bleeding & $9(17)$ & $0(0)$ & $6(12)$ & $0(0)$ \\
\hline Rectal bleeding & $4(8)$ & $0(0)$ & $1(2)$ & $0(0)$ \\
\hline \multicolumn{5}{|l|}{ Gastrointestinal } \\
\hline hemorrhage & $3(6)$ & $0(0)$ & $5(10)$ & $1(2)$ \\
\hline \multicolumn{5}{|l|}{$\begin{array}{l}\text { General disorders and } \\
\text { administration site }\end{array}$} \\
\hline conditions & $11(21)$ & $0(0)$ & $3(6)$ & $0(0)$ \\
\hline Injection site hemorrhage & $9(17)$ & $0(0)$ & $3(6)$ & $0(0)$ \\
\hline Investigations & $9(17)$ & $0(0)$ & $7(14)$ & $0(0)$ \\
\hline Hematuria & $7(13)$ & $0(0)$ & $6(12)$ & $0(0)$ \\
\hline \multicolumn{5}{|l|}{ Respiratory, thoracic, and } \\
\hline mediastinal disorders & $24(46)$ & $1(2)$ & $21(41)$ & $0(0)$ \\
\hline Epistaxis & $22(42)$ & $1(2)$ & $21(41)$ & $0(0)$ \\
\hline Hemoptysis & $6(12)$ & $0(0)$ & $2(4)$ & $0(0)$ \\
\hline \multicolumn{5}{|l|}{ Skin and subcutaneous } \\
\hline tissue disorders & $19(37)$ & $0(0)$ & $15(29)$ & $0(0)$ \\
\hline Purpura & $8(15)$ & $0(0)$ & $7(14)$ & $0(0)$ \\
\hline Petechiae & $7(13)$ & $0(0)$ & $8(16)$ & $0(0)$ \\
\hline Ecchymosis & $3(6)$ & $0(0)$ & $0(0)$ & $0(0)$ \\
\hline \multicolumn{5}{|l|}{ Surgical and medical } \\
\hline procedure & $3(6)$ & $0(0)$ & $1(2)$ & $0(0)$ \\
\hline Postoperative hemorrhage & $3(6)$ & $0(0)$ & $0(0)$ & $0(0)$ \\
\hline Vascular disorders & $7(13)$ & $0(0)$ & $4(8)$ & $0(0)$ \\
\hline Hematoma & $7(13)$ & $0(0)$ & $4(8)$ & $0(0)$ \\
\hline
\end{tabular}

The numbers of patients with hemorrhagic adverse events with a frequency of $5 \%$ or higher are listed for system organ class and for preferred terms within each system organ class. Within some system organ classes individual events (terms) occurred with a frequency of less than $5 \%$; these were not listed but were captured within the system organ class. Some patients had more than one type of hemorrhagic event within a system organ class. The proportions (\%) were compared by $\chi^{2}$ tests to determine whether statistical differences were present between the treatmen groups. The proportion of severe hemorrhagic adverse events is indicated separately. Mild and moderate adverse events did not require specific treatment interven tions. Severe adverse events required treatment intervention and change in patient activity status. There were no statistical differences $(P>.05)$ between the treatment groups with respect to the incidence of any hemorrhagic adverse events. 
provided similar count increments and CCIs. ${ }^{18}$ PRP platelets are stored in $100 \%$ donor plasma as individual concentrates rather than as pools in a mixture of plasma with platelet additive solution. Therefore, PRP platelets prepared with photochemical treatment for pathogen inactivation may potentially demonstrate differences in clinical efficacy. Recently we reported preliminary data from a study of single-donor platelets collected by apheresis, suspended in platelet additive solution, and prepared with photochemical pathogen inactivation. ${ }^{22}$ This study demonstrated that single-donor PCT platelets were equivalent to conventional platelets for prevention and treatment of bleeding in severely thrombocytopenic patients receiving multiple platelet transfusions. However, average 1- and 24-hour platelet count increments were significantly lower in patients supported with single-donor PCT platelets. Thus, different processing methods may result in differences in posttransfusion count increments.

The present study was designed with sufficient power to detect potentially relevant clinical differences in count increments between PCT and conventional platelets. Posttransfusion count increments are surrogate measures of platelet transfusion efficacy used in clinical practice. There are no established standards defining an adequate response to platelet transfusion; however, there is general recognition that a 1-hour CCI of 7500 or more constitutes an adequate response. ${ }^{23}$ Earlier studies of the response to platelet transfusion demonstrated that multiple covariate factors affect the response to platelet transfusion. ${ }^{24-26}$ In addition, the count increment response decreases with multiple transfusions, even in nonalloimmunized patients ${ }^{27}$; thus comparison of average count increment values for patients receiving varying numbers of transfusions may not be sufficiently robust. For this reason, we limited the analysis of average count increment and CCI values to the first 8 transfusions, since most patients were expected to receive at least 8 transfusions. Furthermore, since the CCI is a ratio measure that incorporates platelet dose and patient size, both recognized to affect count increment responses, we used linear regression analysis with count increment as the dependent variable to assess, independently, the effect of platelet dose and patient size among other potential factors, including the number of platelet transfusions used as the longitudinal variable. ${ }^{28}$

Analysis of the 1- and 24-hour posttransfusion platelet counts for all on-protocol study transfusions by longitudinal regression analysis demonstrated no statistically significant effects of PCT on posttransfusion platelet counts. As expected, the platelet dose, age of the platelet component, pretransfusion platelet count, and patient size were highly significant covariate factors for the posttransfusion platelet count and thus the platelet count increment. Although the doses of test and reference platelets were statistically significantly different, both preparations provided doses sufficient for support of thrombocytopenic patients. In 3 of 4 study centers the same numbers of buffy coats were used to produce pooled platelet doses of test and reference products. In one study center, to comply with standard operating procedures, reference pools could be produced from only 4 buffy coats, while test pools were composed of 5 buffy coats. Using linear regression analysis, we examined the effect of dose on count increment response, since platelet doses were determined at time of transfusion for both types of platelets. This analysis confirmed that equal doses of test and reference platelets provided comparable 1- and 24-hour count increments.

Despite platelet losses of approximately $10 \%$ due to the PCT process, the average platelet dose of test product pools was well above $3.0 \times 10^{11}$, a dose commonly recognized as sufficient for transfusion support. ${ }^{29}$ With an integrated processing set, currently observed losses of approximately $8 \%$ are not expected to require use of additional buffy coats to prepare therapeutic doses of PCT platelets. ${ }^{30}$ Importantly, the responses to test and reference platelet concentrates were comparable over the range of platelet doses used in clinical practice, and average test and reference 1- and 24-hour count increment and CCI were within previously reported ranges for similar patient populations. ${ }^{16,17,26}$ In comparison with other recent studies examining the effect of platelet dose on the interval to the next transfusion, ${ }^{17,29}$ test platelets in the present study demonstrated transfusion intervals within reported ranges.

The mean number of on-protocol transfusions and the total dose of platelets transfused during the 56-day transfusion period were higher in the test group; however, this difference was partly accounted for by the higher rate of patient withdrawal from the reference group (10 patients vs 5 patients), resulting in more days of thrombocytopenia in the test group. Withdrawal most frequently was due to enrollment in a stem cell transplantation protocol for additional therapy that did not allow for use of experimental platelet components. These patients were withdrawn from this study by their primary care physicians who remained blinded to platelet transfusion group assignment. The nonrandom distribution between groups appeared to be a chance event. When the number of transfusions during the first period of platelet support was analyzed for patients who were not withdrawn from the study (43 test and 34 reference patients), the average number of platelet transfusions was 5.5 and $4.1(P=.25)$, respectively. For patients who required a second period of platelet support and completed cycle 1, the average number of platelet transfusions for that population (19 test and 12 reference patients) was 3.5 and 4.4, respectively.

Platelet transfusions are given to prevent and to treat bleeding associated with thrombocytopenia. In this study, assessment of hemostasis utilized integration of the pre- and posttransfusion hemostatic evaluations, all reported hemorrhagic adverse events, and red cell use during the entire period of platelet support. Although at least one hemorrhagic adverse event was observed in the majority of patients during the observation period, only 3 patients in the test group and 3 patients in the reference group experienced severe hemorrhagic adverse events. One test patient and 2 reference patients had cerebral hemorrhage resulting in death during the posttransfusion period. Five patients in the reference group and no patients in the test group received antifibrinolytics during the study. This class of medications could have resulted in reduced bleeding in these 5 patients; however, no clear trend for reduced bleeding was detected in this subset of patients. These patients were included in the analysis for all reference group patients. A recent study of single-donor platelets prepared with PCT and conventional single-donor platelets demonstrated equivalence for prevention and treatment of bleeding during profound thrombocytopenia requiring repeated platelet transfusion. ${ }^{22}$

The present study represents an evaluation of platelet transfusion support in a patient population at high risk for bleeding. The data support the conclusion that pooled buffy-coat platelet components treated with the S-59 pathogen inactivation device and stored for up to 5 days were comparable to conventional platelet components for transfusion support of thrombocytopenic patients. Of note, a substantial proportion of both types of platelet products were stored for 4 or more days before transfusion. In this study, the safety profile of PCT platelets was not different from that of conventional platelet components; however, the scope of our study was small. Since it is not feasible to definitively evaluate the safety of PCT platelets in pediatric or pregnant patients during a clinical trial, we conducted preclinical safety studies with pregnant and neonatal animals to address these issues. We detected no toxicity in response to transfusion of S-59 or PCT plasma in these animal 
populations. ${ }^{31}$ Furthermore, in a carcinogenicity study using mice heterozygous for the p53 mutation, S-59 was not carcinogenic at 1000 times the clinical exposure. ${ }^{31}$

Previously reported studies have demonstrated that the PCT technology provides robust inactivation of viruses, bacteria, and leukocytes in platelet components. ${ }^{5,8,9}$ Based on this clinical trial, pooled platelet components prepared with PCT and stored for up to 5 days offer the potential to reduce transfusion-associated infections and inactivate residual contaminating leukocytes using a processing system that is compatible with the current method of preparing therapeutic doses of buffy coat platelets.

\section{Acknowledgments}

The authors wish to acknowledge the substantial contribution of the following collaborating investigators and study personnel, without whose efforts this study could not have been completed: S.
Larsson, M. Vesterinen, M. Christenson (Huddinge University Hospital, Karolinska Institute, Stockholm, Sweden); R. Evely, D. Marks (Avon Haematology Unit, Bristol, United Kingdom); C. Waller, P. Dufour (EFS-Alsace, Strasbourg, France); C. Oprea, A. Faradji (Department of Oncohematology, Hopital de Hautepierre, Strasbourg, France); and N. Goulden (Department of Haematology and BMT, Bristol Royal Hospital for Sick Children, Bristol, United Kingdom). Lindy Kauffman (Cerus Corporation, Concord, CA) developed the assay for detection of antibodies against potential S-59-associated neoantigens.

\section{Appendix}

The S-59 Platelet Recovery in Thrombocytopenia-Europe (euroSPRITE) investigator group consisted of: Dick van Rhenen, Hans Gulliksson, Jean-Pierre Cazenave, Derwood Pamphilon, Per Ljungman, and Harald Klüter.

\section{References}

1. Schreiber GB, Busch MP, Kleinman SH, Korelitz JJ. The risk of transfusion- transmitted viral infections. N Engl J Med. 1996;334:1685-1690.

2. Ling AE, Robbins KE, Brown TM, et al. Failure of routine HIV-1 tests in a case involving transmission with preseroconversion blood components during the infectious window period. JAMA. 2000; 284:238-240.

3. Schuttler GC, Caspari G, Jursch CA, Willems WR, Gerlich WH, Schaefer S. Hepatitis C virus transmission by a blood donation negative in nucleic acid amplification tests for viral RNA. Lancet. 2000;355:41-42.

4. McDonald CP, Hartley S, Orchard K, et al. Fatal Clostridium perfringens sepsis from a pooled platelet transfusion. Transfusion Med. 1998:8:19-22.

5. Lin L, Cook DN, Wiesehahn GP, et al. Photochemical inactivation of viruses and bacteria in platelet concentrates by use of a novel psoralen and long-wavelength ultraviolet light. Transfusion. 1997;37:423-435.

6. Alfonso R, Lin C, Dupuis K, et al. Inactivation of viruses with preservation of coagulation function in fresh frozen plasma. Blood. 1996;88(suppl 1):526a.

7. Lin L, Corten L, Murthy KK, Corash L, Alter HJ. Photochemical inactivation of hepatitis B (HBV) and hepatitis $\mathrm{C}(\mathrm{HCV})$ virus in human platelet concentrates as assessed by a chimpanzee infectivity model. Blood. 1998:92(suppl 1):502a.

8. Knutson F, Alfonso R, Dupuis K, et al. Photochemical inactivation of bacteria and HIV in buffycoat-derived platelet concentrates under conditions that preserve in vitro platelet function. Vox Sanguinis. 2000;78:209-216.

9. Grass JA, Hei DJ, Metchette K, et al. Inactivation of leukocytes in platelet concentrates by psoralen plus UVA. Blood. 1998;91:2180-2188.

10. Grass JA, Wafa T, Reames A, et al. Prevention of transfusion-associated graft-versus-host disease by photochemical treatment. Blood. 1999;93 3140-3147.

11. van Rhenen DJ, Vermeij J, Mayaudon V, Hind C, Lin L, Corash L. Functional characteristics of
S-59 photochemically treated platelet concentrates derived from buffy coats. Vox Sanguinis. 2000;79:206-214.

12. Brown EG, Wood L, Wood $S$. The medical dictionary for regulatory activities (MedDRA). Drug Saf. 1999;2:109-117.

13. Gmur J, Burger J, Schanz U, Fehr J, Schaffner A Safety of a stringent prophylactic platelet transfusion policy for patients with acute leukemia. Lancet. 1991;338:1223.

14. TRAP Study Group. Leukocyte reduction and ultraviolet B irradiation of platelets to prevent alloimmunization and refractoriness to platelet transfusions. N Engl J Med. 1997;337:1861-1869.

15. Liang KY, Zeger SL. Longitudinal data analysis using generalized linear models. Biometrika. 1986;73:13-22.

16. Sweeney JD, Kouttab NM, Penn CL, McHugh $\mathrm{KE}$, Nelson EJ, Oblon DJ. A comparison of prestorage WBC-reduced whole-blood-derived platelets and bedside-filtered whole-blood-derived platelets in autologous progenitor cell transplant. Transfusion. 2000:40:794-800.

17. Klumpp TR, Herman JH, Gaughan JP, et al. Clinical consequences of alterations in platelet transfusion dose: a prospective randomized, doubleblind trial. Transfusion. 1999;39:674-681.

18. van Rhenen DJ, Vermeij J, Kappers-Klunne MC Payrat JM. Evaluation of a new citrate-acetate$\mathrm{NaCl}$ platelet additive solution for the storage of white cell reduced platelet concentrates obtained from half strength CPD pooled buffy coats. Transfusion. 1995;35:50-53.

19. Murphy S, Heaton WA, Rebulla P. Platelet production in the Old World-and the New. Transfusion. 1996;36:751-754.

20. Bertolini F, Rebulla P, Riccardi D, Cortellaro M, Ranzi ML, Sirchia G. Evaluation of platelet concentrates prepared from buffy coats and stored in glucose-free crystalloid medium. Transfusion. 1989;29:605-609.

21. Eriksson L, Kristensen J, Olsson J. Evaluation of platelet function using the in vitro bleeding time and corrected count increment of transfused platelets. Comparison between platelet concentrates derived from pooled buffy coats and apheresis. Vox Sanguinis. 1996;70:69-75.

22. McCullough J, Vesole D, Benjamin RJ, et al. Pathogen inactivated platelets (plt) using Helinx technology (INTERCEPT plt) are hemostatically effective in thrombocytopenic patients (tcp pts): the SPRINT trial. Blood. 2001;98(suppl 1):450a

23. Leach MF, AuBuchon JP. Effect of storage time on clinical efficacy of single-donor platelet units. Transfusion. 1993;33:661-664.

24. Bishop JF, Matthews JP, McGrath K, Yuen K, Wolf MM, Szer J. Factors influencing 20-hour increments after platelet transfusion. Transfusion. 1991;31:392-396.

25. Bishop JF, McGrath K, Wolf MM, et al. Clinical factors influencing efficacy of pooled platelet transfusions. Blood. 1988;71:383-387.

26. Ishida A, Handa M, Wakui M, Okamoto S, Kamakura M, Ikeda Y. Clinical factors influencing posttransfusion platelet increment in patients undergoing hematopoietic progenitor cell transplantation-a prospective analysis. Transfusion. 1998; 38:839-847.

27. Enright $\mathrm{H}$, Gernsheimer T, Woodson R, et al. Factors influencing the response to platelet transfusion. Blood. 1997;90(suppl 1):268a

28. Davis KB, Slichter SJ, Corash L. Corrected count increments and platelet recoveries as measures of post-transfusion platelet response: problems and a solution. Transfusion. 1999;39:586-592.

29. Norol F, Bierling P, Roudot-Thoraval F, et al. Platelet transfusion: a dose-response study. Blood. 1998;92:1448-1453.

30. Vermeij J, Mayaudon V, Flament J, et al. The INTERCEPT platelet system for pathogen inactivation with an integral processing set demonstrated improved process control for production of platelet concentrates. Vox Sanguinis. 2002;83(suppl 2):110.

31. Ciaravino V. Preclinical safety of a nucleic acidtargeted helinx compound: a clinical perspective. Semin Hematol. 2001;38(suppl 11):12-19. 